CLINICAL STUDY

\title{
Free leptin index and thyroid function in male highly trained athletes
}

\author{
Gianluca Perseghin $^{1,2}$, Guido Lattuada ${ }^{2}$, Francesca Ragogna $^{2}$, Giampietro Alberti ${ }^{1}$, Antonio La Torre ${ }^{1}$ \\ and Livio Luzi ${ }^{1,2}$ \\ ${ }^{1}$ Department of Sport, Nutrition and Health, Università degli Studi di Milano, via Kramer 4/a 20129 Milan, Italy and ${ }^{2}$ Internal Medicine, San Raffaela \\ Scientific Institute, via Olgettina 60, 20132 Milan, Italy
}

(Correspondence should be addressed to G Perseghin at Department of Sport, Nutrition and Health, Università degli Studi di Milano and San Raffaele Scientific Institute, Internal Medicine; Email: perseghin.gianluca@hsrit)

\begin{abstract}
Objective: Exercise training may cause changes in thyroid function. This thyroid response may be due to exercise-induced modulation of energy metabolism but also of the adipocytes endocrine function. In particular, the role of leptin and of circulating soluble leptin receptor (sOB-R) was unexplored. The aim of this study was to assess the relationships between thyroid function, whole body energy metabolism, and adipokines - mainly leptin and its receptor, sOB-R.

Methods: We measured serum TSH, free tri-iodothyronine $\left(\mathrm{FT}_{3}\right)$, free thyroxine, leptin, and sOB-R and assessed energy homeostasis by means of indirect calorimetry, in 27 highly trained athletes and 27 sedentary, healthy men.

Results: TSH-FT 3 ratio was lower in athletes $(P<0.03)$, either in sustained power or anaerobic powersprint athletes $(n=13)$ or marathon runners $(n=14)$. Whole body respiratory quotient was lower in athletes. Fasting serum sOB-R was higher and leptin lower in athletes than controls. Also serum adiponectin, resistin, and retinol binding protein- 4 concentrations were different in athletes than in controls. The ratio between leptin and sOB-R, the free leptin index (FLI), was lower in athletes than in controls $(0.025 \pm 0.014$ vs $0.085 \pm 0.049 ; P<0.001)$. In multivariate analysis, FLI retained independent association with $\mathrm{TSH}-\mathrm{FT}_{3}$ ratio.

Conclusion: Male, elite athletes had lower $\mathrm{TSH}-\mathrm{FT}_{3}$ ratio and FLI than controls while FLI was independently associated with $\mathrm{TSH}-\mathrm{FT}_{3}$ ratio supporting the hypothesis that the level of biologically active leptin is involved in the adaptive response of thyroid function in professional athletes.
\end{abstract}

European Journal of Endocrinology $161871-876$

\section{Introduction}

Regulation of the metabolic adaptation during prolonged physical training by the thyroid hormones is considered of importance but remains controversial. Some authors reported no major effect of exercise training on serum TSH levels in professional athletes (1) and sedentary individuals (2) suggesting that the influence of chronic exercise is likely to be small; in contrast other authors suggested long-term exercise training may lead to downregulation of thyroid hormone concentrations (3-5). Among the candidate factors, this response may be induced by the exerciseinduced negative energy balance as well as signaling alterations in the hypothalamus-pituitary-thyroid and hypothalamus-adipocyte-leptin axes (5). With this respect, leptin is an adipocyte-secreted hormone that plays an important role as a signal of energy availability in energy-deficient states (6), and during training situations both leptin and TSH levels were shown to decrease in parallel (7).

In this context, our study aimed to assess simultaneously whole body energy metabolism, thyroid function and circulating leptin concentration in professional, elite track runners and to compare them to sedentary age- and body mass index (BMI)-matched subjects. Leptin may bind to several membrane-bound isoforms, but a soluble form of the leptin receptor (sOB-R) is also generated by cleavage of the membranebound isoform (8). sOB-R is therefore an important regulator of leptin activity (9). The balance between the serum leptin and sOB-R concentration, known as free leptin index (FLI), is the ratio between the two concentrations and was recently reported to be associated with metabolic features in insulin resistant subjects (10). For this reason we decided to test whether the habitual, long lasting habit to perform heavy physical activity was associated not only with changes 
in the serum leptin levels, but also with its soluble receptor and with FLI. We also measured in the same population the serum concentration of other adipokines presumably involved in the regulation of energy metabolism and insulin action such as adiponectin, resistin, retinol binding protein-4 (RBP-4), and visfatin.

\section{Methods}

\section{Subjects}

Twenty-seven elite track runners (sustained power or anaerobic power-sprint athletes $n=13$; marathon runners $n=14$ ) participating in national and international competitions were recruited within the Federazione Italiana Atletica Leggera Lombardia Section. They were compared with 27 sedentary young lean men. Matching of age and BMI was 1:1 and was performed within five units of age and one unit of BMI. These healthy individuals were recruited in the outpatient services of the Center of Nutrition/Metabolism of the San Raffaele Scientific Institute. Body weight was stable for at least 6 months. The clinical and laboratory features of the subjects are summarized in Table 1. All subjects were in good health as assessed by medical history, physical examination, hematological, and urinalysis. Informed consent was obtained from all subjects after explanation of purposes, nature, and

Table 1 Anthropometric, laboratory, and metabolic characteristics of study subjects.

\begin{tabular}{|c|c|c|}
\hline & Athletes & Normals \\
\hline Number & 27 & 27 \\
\hline Age (years) & $28 \pm 11$ & $30 \pm 6$ \\
\hline Body weight (kg) & $68 \pm 7$ & $68 \pm 5$ \\
\hline Height $(\mathrm{cm})$ & $178 \pm 6$ & $177 \pm 6$ \\
\hline BMI $\left(\mathrm{kg} / \mathrm{m}^{2}\right)$ & $21.5 \pm 1.1$ & $21.8 \pm 1.2$ \\
\hline Body fat (\%) & $8.1 \pm 0.4^{*, a}$ & $11.6 \pm 0.8$ \\
\hline Plasma glucose (mg/dl) & $85 \pm 7$ & $85 \pm 7$ \\
\hline Serum insulin $(\mu \mathrm{U} / \mathrm{ml})$ & $7.7 \pm 4.2^{\dagger}$ & $10.6 \pm 4.9$ \\
\hline Serum C-peptide (ng/ml) & $1.24 \pm 0.65^{*}$ & $1.96 \pm 0.91$ \\
\hline HOMA2-\%S & $113 \pm 51^{\dagger}$ & $83 \pm 41$ \\
\hline HOMA2-\%B & $114 \pm 42^{\dagger}$ & $143 \pm 55$ \\
\hline Serum FFA (mmol/l) & $0.63 \pm 0.24$ & $0.53 \pm 0.22$ \\
\hline Total cholesterol (mg/dl) & $152 \pm 38$ & $164 \pm 31$ \\
\hline HDL-cholesterol (mg/dl) & $57 \pm 10$ & $55 \pm 13$ \\
\hline Triglycerides (mg/dl) & $62 \pm 22$ & $72 \pm 38$ \\
\hline hsČRP (mg/dl) & $1.11 \pm 0.96$ & $0.82 \pm 0.77$ \\
\hline Free $T_{3}(p g / m l)$ & $5.4 \pm 0.9$ & $5.3 \pm 0.9$ \\
\hline Free $\mathrm{T}_{4}(\mathrm{pg} / \mathrm{ml})$ & $18.2 \pm 3.9$ & $18.5 \pm 3.4$ \\
\hline $\mathrm{TSH}(\mu \mathrm{U} / \mathrm{ml})$ & $0.90 \pm 0.65^{\dagger}$ & $1.27 \pm 0.76$ \\
\hline $\mathrm{TSH} /$ free $_{3}$ ratio & $0.16 \pm 0.11^{\dagger}$ & $0.25 \pm 0.17$ \\
\hline REE (kcal/die) & $1903 \pm 324$ & $1796 \pm 214$ \\
\hline $\begin{array}{l}\text { Percentage of predicted } \\
\text { REE (\%) }\end{array}$ & $112 \pm 16$ & $107 \pm 11$ \\
\hline$R Q$ & $0.77 \pm 0.06^{\dagger}$ & $0.83 \pm 0.11$ \\
\hline
\end{tabular}

${ }^{*} P<0.01$ versus normals; ${ }^{\dagger} P<0.05$ versus normals (2-tails independent $t$-test). REE, resting energy expenditure; $R Q$, respiratory quotient.

${ }^{a}$ Assessment of body fat was obtained in 19 athletes. potential risks of the study. The protocol was approved by the Ethical Committee of the San Raffaele Scientific Institute.

\section{Experimental protocol}

Subjects were instructed to consume an isocaloric diet (containing at least $250 \mathrm{~g}$ carbohydrates and 70-90 g protein/day) and to abstain from exercise activity for 3 days before the studies. Subjects were admitted to the Metabolic Room of the Section of Nutrition/Metabolism of the San Raffaele Scientific Institute at $0730 \mathrm{~h}$ after a 10-h period overnight fast. Indirect calorimetry was performed continuously, while lying quietly, for $30 \mathrm{~min}$ with a ventilated hood system (Sensor Medics 2900, Metabolic Measurement Cart, Milano, Italy). The mean coefficients of variation $(\mathrm{CV})$ within the session for both $\mathrm{O}_{2}$ and $\mathrm{CO}_{2}$ measurements were below $3 \%$. Subsequently, a blood sample was obtained for the assessment of fasting serum glucose, insulin, free fatty acids (FFA), total cholesterol, high density lipoprotein (HDL)-cholesterol, triglycerides, leptin, sOB-R, adiponectin, resistin, visfatin, $\mathrm{RBP}-4$, high sensitivity $\mathrm{C}$ reactive protein (hsCRP), $\mathrm{TSH}$, free tri-iodothyronine $\left(\mathrm{FT}_{3}\right)$, and free thyroxine $\left(\mathrm{FT}_{4}\right)$. Body fat was assessed the morning prior to the indirect calorimetry by means of bioelectrical impedance using a Body Impedance Analyzer (Akem, RIL System, Detroit, MI, USA) in the 27 control subjects and in 19 athletes.

\section{Analytical determinations}

Glucose concentration was measured with standard glucose oxidase method on a glucose analyzer (Beckman Glucose Analyzer 2, Inc., Fullerton, CA, USA). Blood samples for FFA assessment were collected in prechilled tubes containing $0.1 \%$ EDTA. Tubes were immediately placed in ice and plasma was immediately processed by centrifugation at $4{ }^{\circ} \mathrm{C}$. Plasma was then frozen and stored at $-70{ }^{\circ} \mathrm{C}$ and FFA determinations were performed within 0-5 working days as suggested by Zambon et al. to obtain plasma FFA determination (microfluorometric assay) avoiding lipoprotein lipase reaction in blood (11). Serum triglycerides, total cholesterol, and high density lipoprotein (HDL)-cholesterol were measured as previously described (enzymatic method on an Hitachi 747) (12). Low density lipoprotein-cholesterol was calculated using the Friedewald formula. Serum resistin was measured by ELISA kit (BioVendor Laboratory Medicine, Inc., Brno, Czech Republic). The sensitivity of the assay was $0.2 \mathrm{ng} / \mathrm{ml}$ of sample. The intra-assay $\mathrm{CV}$ was $<3.5 \%$ and inter-assay was $<7 \%$. Serum levels of insulin (sensitivity $2 \mu \mathrm{U} / \mathrm{ml}$; intra- and interassay $\mathrm{CV}<3.1$ and $6 \%$ respectively) and leptin (sensitivity $0.5 \mathrm{ng} / \mathrm{ml}$; intra- and interassay $\mathrm{CV}<5$ and $9 \%$ respectively) were measured with RIA (Linco Research, St Charles, MO, USA) following the manufacturer's assay protocols. Serum adiponectin was 
measured by ELISA kit (B-Bridge International, Inc., Sunnyvale, CA, USA) with a sensitivity of $25 \mathrm{pg} / \mathrm{ml}$. Serum high sensitive $C$-reactive protein was measured using an ELISA kit (Diagnostic Biochem Canada Inc., London, Ontario, Canada) with a sensitivity of $10 \mathrm{ng} / \mathrm{ml}$ and intra- and interassay $\mathrm{CV}<5$ and $<7 \%$ respectively. Serum sOB-R was measured by ELISA kit (BioVendor Laboratory Medicine, Inc.) with a sensitivity of $0.04 \mathrm{ng} / \mathrm{ml}$ and intra- and interassay $\mathrm{CV}<7$ and $<10 \%$ respectively. Serum $\mathrm{FT}_{3}$ (sensitivity $0.3 \mathrm{pg} / \mathrm{ml}$; intra- and interassay $\mathrm{CV}<3.5$ and $9 \%$ respectively) and $\mathrm{FT}_{4}$ (sensitivity $1 \mathrm{pg} / \mathrm{ml}$; intra- and interassay $\mathrm{CV}<5$ and $10 \%$ respectively) were measured with ELISA (Diagnostic Biochem Canada Inc.) following the manufacturer recommendations. TSH was measured by immunofluorimetric method. Finally, urine nitrogen was measured by the Kjedhal method as previously reported (13).

\section{Calculations}

Insulin sensitivity was estimated by means of the updated computer model homeostasis model assessment (HOMA)-2 indexes (14) available from www.OCDEM.ox. ac.uk. Resting energy expenditure (REE) was calculated by the Weir's standard equation from the oxygen consumption rate and the carbon dioxide production rates measured by means of indirect calorimetry (excluding the first $10 \mathrm{~min}$ of data acquisition), and from the urinary nitrogen excretion. Predicted REE was calculated using the Harris-Benedict equations (15). The percentage of predicted REE was calculated as the ratio predicted to be measured (16). The balance between leptin and sOB-R, the FLI, was determined by calculating the ratio between the serum concentrations of leptin and sOB-R as previously described (10).

\section{Statistical analysis}

Data in text, tables, and figures are mean \pm s.D. Analysis was performed using the SPSS software (version 13.0; SPSS Inc., Chicago, IL, USA). When parameters (HOMA\%-IS, serum triglycerides) showed a skewed distribution (Kolmogorov-Smirnov test of normality), they were log-transformed before the analysis. Comparison between groups was performed using 2-tailed independent samples $t$-test or Kruskal-Wallis nonparametric test depending on the distribution of the data, and a $P$ value $<0.05$ was considered to be statistically significant. Two-tailed Pearson's correlation was performed to establish partial correlation coefficients between variables and also in this case, nonparametric correlation coefficient was obtained using Spearman's $\rho$ when appropriate. To ascertain the relative contribution of independent factors on the $\mathrm{TSH}-\mathrm{FT}_{3}$ ratio, we used stepwise regression analysis (using $F$ ratio-to-remove of 4 and $F$ ratio-to-enter of 3.996) including the parameters that in univariate analysis that had a $P$ value $<0.1$.

\section{Results}

\section{Clinical features}

Anthropometric and laboratory features of the elite athletes and controls are summarized in Table 1. The two study groups were not different for age and BMI but athletes had a lower body fat mass. Fasting serum glucose concentration was also not different, while serum insulin and C-peptide concentrations were lower in athletes than in the control group. As a consequence, the surrogate index of insulin sensitivity based on fasting glucose and insulin concentration (HOMA2-\%S) was higher in athletes than controls and the surrogate index of $\beta$-cell sensitivity (HOMA2-\%B) was lower in athletes than controls. With respect to lipid metabolism the lipoprotein profile was not different between groups as well as the fasting serum FFA concentration $(P=0.102)$. hsCRP, as a surrogate marker of low-grade inflammation was not different between groups $(P=0.45)$.

\section{Parameters of thyroid function}

Parameters of thyroid function are summarized in Table 1. Fasting serum $\mathrm{FT}_{3}$ and $\mathrm{FT}_{4}$ concentrations were not different in athletes in comparison to sedentary. Serum TSH was lower in athletes when compared to sedentary as well as the $\mathrm{TSH} / \mathrm{FT}_{3}$ ratio.

\section{Whole body energy metabolism}

Parameters of whole body energy metabolism are summarized in Table 1. REE rate and percentage of predicted REE were not different between groups. The respiratory quotient (RQ) was lower in athletes when compared to sedentary. REE corrected for the kg of LBM was also not different between groups $(30.5 \pm 2.0$ vs $29.9 \pm 1.1 \mathrm{kcal} / \mathrm{kg}$ of $\mathrm{LBM} ; P=0.201)$.

\section{Adipokines concentration}

Fasting serum adipokines concentrations are summarized in Fig. 1. Fasting serum leptin concentration was lower in athletes than sedentary $(1.39 \pm 0.51$ vs $3.33 \pm 1.50 \mathrm{ng} / \mathrm{ml} ; P=0.001)$ meanwhile sOB-R was higher in the athletes than sedentary $(59 \pm 13$ vs 47 $\pm 20 \mathrm{ng} / \mathrm{ml} ; \quad P=0.019$ ). The balance between leptin and sOB-R, the FLI was lower in athletes than in sedentary $(0.025 \pm 0.014$ vs $0.085 \pm 0.049$; $P<0.001)$. Serum adiponectin $(8.4 \pm 2.9$ vs 6.4 $\pm 2.8 \mu \mathrm{g} / \mathrm{ml} ; \quad P=0.014)$ and resistin $(4.3 \pm 0.8$ vs. $3.7 \pm 1.1 \mathrm{ng} / \mathrm{ml} ; \quad P=0.048)$ were higher in athletes than sedentary. RBP-4 was lower in athletes than in sedentary $(30 \pm 7$ vs $34 \pm 7 \mu \mathrm{g} / \mathrm{ml} ; P=0.025)$ meanwhile serum visfatin $(15 \pm 9$ vs $12 \pm 5 \mathrm{ng} / \mathrm{ml} ; P=0.28)$ was not different between groups. 

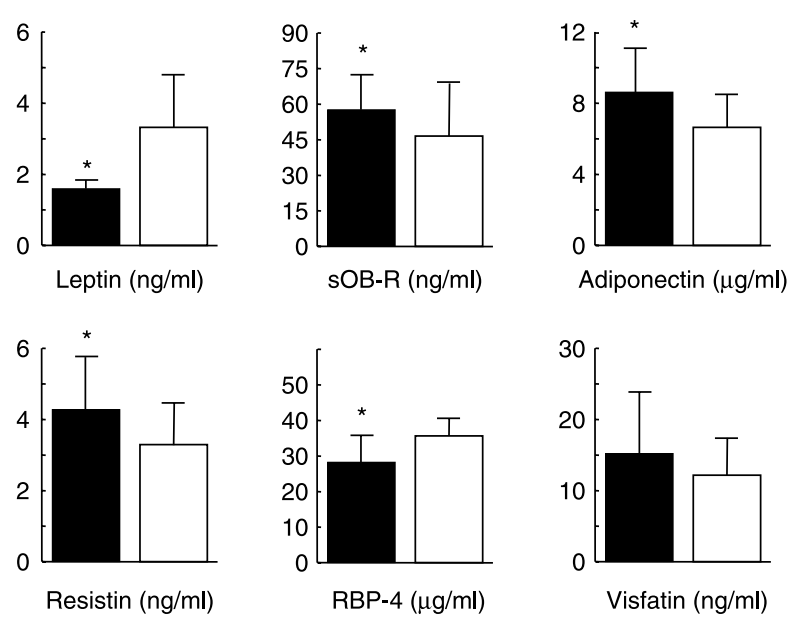

Figure 1 Serum adipokines and SOB-R concentrations in study groups. Fasting serum concentrations of leptin, sOB-R, adiponectin, resistin, RBP-4, and visfatin are summarized. Black columns represent the athletes, white columns the sedentary control subjects. Mean \pm s.D. ${ }^{*} P<0.05$ versus normal sedentary controls (two-tailed independent samples $t$-test).

\section{Correlation analysis}

The age- and BMI-adjusted Pearson's correlation showed that among the parameters of energy metabolism the $\mathrm{TSH} / \mathrm{FT}_{3}$ ratio correlated with $\mathrm{RQ}$, and showed a trend with the percentage of predicted REE (Table 2). The analysis showed a significant correlation with the sOB-R and FLI, but not with leptin, adiponectin, resistin, retinol-binding protein 4 , and visfatin (Table 2). The $\mathrm{TSH} / \mathrm{FT}_{3}$ ratio was also significantly associated with fasting serum triglycerides, and showed a trend with HDL-cholesterol and fasting serum insulin (Table 2). No significant association was detected with fasting serum glucose, C-peptide, FFA, and hsCRP concentrations. There was clearly no significant correlation between the $\mathrm{TSH} / \mathrm{FT}_{3}$ ratio and fat mass in the two subgroups separately and when the correlation analysis was performed in the entire cohort (fat mass was available in only 19 of the 27 elite athletes) a significant association remained undetectable $(r=-0.21 ; P=0.201)$; nevertheless we cannot exclude that a hypothetical analysis with all the athletes included in the plot could be able to generate a trend for a significant association.

As summarized in Table 2, when stepwise regression analysis model including the independent variables with $P$ value $<0.1$ in univariate analysis was performed, FLI and serum triglycerides retained independent association with the $\mathrm{TSH} / \mathrm{FT}_{3}$ ratio.

\section{Discussion}

It is believed that long-term overtraining may lead to downregulation of hypothalamic hormonal responses; in particular in professional athletes adaptation of the thyroid function may be secondary to the catabolic training condition and/or hormonal feedback from the depleted adipose tissue, with leptin acting as a major endocrine modulator.

In this background, our study is the first to explore the relationship between parameters of thyroid function and leptin taking simultaneously into account the serum soluble leptin circulating receptor (sOB-R) concentrations in male, elite, professional athletes. We found that adaptation of the thyroid function was not only in parallel with lower serum leptin concentrations but also with higher sOB-R and that the FLI was independently associated with the thyroid function. In these subjects we also assessed the serum levels of other adipokines, driving us to the conclusion that even if their levels may be also different than in sedentary, they are not associated as tightly as FLI with the thyroid function.

The impact of exercise on thyroid hormone metabolism is somewhat controversial as well as the impact on leptin and other adipokines (17). In longitudinal studies, this was probably due to differences in the intensity, duration, and form of exercise as well as on the baseline individual fitness and metabolic conditions and on gender (18). For this reason we decided to explore the issue limiting initially our focus on a group of male, elite professional (long lasting involvement in heavy exercise training program) athletes and comparing them to a control group of sedentary normal-weight subjects. We found that in the fasting and resting state they had a subtle but significant lower serum TSH level which was more evident when the TSH level was normalized to the $\mathrm{FT}_{3}$ (Table 1).

We measured resting whole body energy metabolism by means of indirect calorimetry and found that it was not different in athletes than in the sedentary controls. This finding is in agreement with a work by Befroy et al. in which resting energy metabolism was assessed by means of 31P-magnetic resonance spectroscopy (MRS) of the skeletal muscle (19). Correlatively we noticed that the fasting oxidative metabolism of these athletes was shifted towards fatty acids oxidation as reflected by the lower RQ (Table 1). The percentage of predicted REE and the RQ were correlatively associated with the TSH-FT 3 ratio (Table 1). This association in univariate analysis was not retained when the multivariate analysis was performed.

An additional aim of the present work was to evaluate the relationship among the thyroid function and serum leptin not in isolation, as generally reported, but taking simultaneously into consideration the serum levels of other adipokines also known to be potentially involved in the regulation of insulin sensitivity, body fat distribution, and energy metabolism. Not surprisingly, we found that serum leptin was lower in the athletes and adiponectin was higher in athletes due to higher insulin sensitivity (as reflected by the higher HOMA2\%-S). Serum resistin was lower as we have 
Table 2 Results of the correlation and multiple regression analysis between $\mathrm{TSH} /$ free tri-iodothyronine $\left(\mathrm{FT}_{3}\right)$ ratio, metabolic parameters, and serum adipokines concentrations.

\begin{tabular}{|c|c|c|c|c|}
\hline & Variable & $r$ & $\beta$ & $P$ \\
\hline \multicolumn{5}{|c|}{ Partial correlation analysis (age-, and BMI-adjusted) } \\
\hline \multirow[t]{7}{*}{ Metabolic parameters } & Triglycerides & 0.46 & _ & 0.001 \\
\hline & $\mathrm{RQ}$ & 0.32 & - & 0.037 \\
\hline & Percentage of predicted REE & -0.28 & - & 0.073 \\
\hline & HDL-cholesterol & -0.25 & - & 0.076 \\
\hline & Insulin & 0.24 & - & 0.094 \\
\hline & HOMA2-\%S & -0.13 & _- & 0.41 \\
\hline & Glucose & -0.07 & - & 0.64 \\
\hline \multirow[t]{7}{*}{ sOB-R, FLI and adipokines } & sOB-R & -0.44 & - & 0.003 \\
\hline & FLI & 0.40 & - & 0.004 \\
\hline & Leptin & 0.25 & - & 0.11 \\
\hline & Resistin & 0.23 & - & 0.14 \\
\hline & Adiponectin & -0.21 & - & 0.19 \\
\hline & Visfatin & 0.07 & _- & 0.67 \\
\hline & Retinol binding protein 4 & -0.00 & - & 0.98 \\
\hline \multicolumn{5}{|c|}{$\begin{array}{l}\text { Stepwise multiple regression analysis } \\
\text { (including age, BMl, and dependent variables } \\
\text { with } P<0.1 \text { in univariate analysis) }\end{array}$} \\
\hline \multirow{9}{*}{$\begin{array}{l}\text { Step } 1 \\
\text { Step } 2 \\
\text { Excluded variables at step } 2\end{array}$} & FLI & 0.46 & 0.46 & 0.001 \\
\hline & FLI+triglycerides & 0.56 & $0.36 / 0.33$ & 0.001 \\
\hline & $S O B-B$ & -027 & -030 & 0061 \\
\hline & Age & -0.25 & -0.21 & 0.086 \\
\hline & Percentage of predicted REE & -0.22 & -0.19 & 0.13 \\
\hline & HDL-cholesterol & -0.14 & -0.12 & 0.35 \\
\hline & BMI & -0.05 & 0.05 & 0.73 \\
\hline & $\mathrm{RQ}$ & -0.03 & -0.03 & 0.83 \\
\hline & Insulin & -0.02 & -0.02 & 0.88 \\
\hline
\end{tabular}

$r$, the correlation coefficient; $\beta$, the standardized regression coefficient. Bold indicates $P<0.05$.

previously reported (12) as well as the RBP4 as reported by others (20). In the present work we report two novel findings in this area; serum visfatin was not different between the athletes and sedentary subjects (Fig. 1) and most importantly sOB-R was higher in the athletes (Fig. 1). The leptin receptor can be spliced into different isoforms but the soluble form, sOB-R is of particular interest because it constitutes the secretory (soluble) form of the receptor which is directly released into the circulation where, by binding leptin, it controls leptin bioavailability (9). Only very recently has its importance been taken into consideration when the balance between the serum leptin and sOB-R concentration, known as FLI, was reported to be associated with a number of features of the metabolic syndrome cross-sectionally (10). With respect to all the above-described adipokines, only the FLI retained an independent association when tested in the multivariate, stepwise regression analysis. This finding would suggest that sOB-R and leptin share an important combined endocrine effect in modulating the thyroid function in regularly exercising human subjects. Along with FLI the fasting serum triglycerides concentration also retained an independent association with the thyroid function in the stepwise regression analysis, recalling that signaling from metabolic surrogate markers of the nutritional status are also relevant in regulating the thyroid function in energy deprivation and catabolic conditions such as intense exercise training programs.

An important limitation of the present work is the lack of the assessment of the fitness level in a quantitative fashion. The collection of $\mathrm{VO}_{2 \max }$ data, for example, would have allowed us to establish whether the adaptation of the $\mathrm{TSH} / \mathrm{FT}_{3}$ ratio and more importantly of FLI could be significantly related to the fitness level making our observation more robust. In addition a more detailed analysis of body fat distribution using techniques able to give insights also about topography of body fat could be useful to further clarify the relationships between the metabolic and endocrine changes in the athletes which were characterized in general terms by a lower body fat mass. In particular, the knowledge of the waist circumference as a surrogate marker of visceral adiposity could have been useful to establish whether the changes in leptin and sOB-R observed in the athletes were directly linked to the exercise habit of these individuals or whether they were secondary to the different and visceral adiposity. This suspect could be generated by the observation that in the stepwise multiple regression analysis also serum triglycerides concentration (as a potential marker reflecting the amount of visceral adiposity) fitted the model along with the FLI (Table 2).

These data represent the first report suggesting a potential role for the circulating bioavailable leptin, 
rather than leptin per se, in exercise physiology. Additional studies are warranted to firmly establish not only a role for FLI as a surrogate biomarker of adaptation to exercise but also as a surrogate biomarker in other metabolic conditions associated with energy deprivation and excess, as well as insulin resistance and cardiovascular disease. For example the effects of the acute performance of physical exercise and also of the long-term exercise training program, not only in fit individuals but also in insulin resistant states, should be established.

In conclusion, this study demonstrated that exerciseinduced parameters of thyroid function in male, elite professional athletes were independently associated with the FLI which is based on the fasting serum leptin and sOB-R concentrations, supporting the hypothesis that leptin bioavailability rather than the crude serum leptin concentration is involved in the adaptive response of thyroid function in professional athletes.

\section{Declaration of interest}

There is no conflict of interest that could be perceived as prejudicing the impartiality of the research reported.

\section{Funding}

This study was supported by grants by the Italian Minister of Health (RF98.49, RF99.55, and RF01.1831) and by European Community's FP6. EWA (LSHM-CT-2005-518245).

\section{References}

1 Wesche MF \& Wiersinga WM. Relation between lean body mass and thyroid volume in competition rowers before and during intensive physical training. Hormone and Metabolic Research 2001 33 423-427.

2 Kyriazis GA, Caplan JD, Lowndes J, Carpenter RL, Dennis KE, Sivo SA \& Angelopoulos TJ. Moderate exercise-induced energy expenditure does not alter leptin levels in sedentary obese men. Clinical Journal of Sport Medicine 200717 49-51.

3 Poehlman ET, Tremblay A, Nadeau A, Dussault J, Theriault G \& Bouchard C. Heredity and changes in hormones and metabolic rates with short-term training. American Journal of Physiology. Endocrinology and Metabolism 1986250 E711-E717.

4 Hilton LK \& Loucks AB. Low energy availability, not stress, suppresses diurnal rhythm of leptin in healthy young women. American Journal of Physiology. Endocrinology and Metabolism 2000 278 E43-E49.

5 Steinacker JM, Brkic M, Simsch M, Nething K, Kresz A, Prokopchuk O \& Liu Y. Thyroid hormones, cytokines, physical training and metabolic control. Hormone and Metabolic Research $200537538-544$.

6 Chan JI \& Mantzoros CS. Role of leptin in energy-deprivation states: normal human physiology and clinical implications for hypothalamic amenorrhoea and anorexia nervosa. Lancet 2005 $36674-85$.
7 Simsch C, Lormes W, Petersen KG, Baur S, Liu Y, Hackney AC, Lehamnn M \& Steinacker JM. Training intensity influences leptin and thyroid hormones in highly trained rowers. International Journal of Sports Medicine 200223 422-427.

8 Maamra M, Bidlingmaier M, Postel-Vinay MC, Wu Z, Strasburger CJ \& Ross RJ. Generation of human soluble leptin receptor by proteolytic cleavage of membrane anchored receptors. Endocrinology 2001142 4389-4393.

9 Zastrow O, Seidel B, Kiess W, Thiery J, Keller E, Bottner A \& Kratzsc J. The soluble letin receptor is crucial for leptin action: evidence from clinical and experimental data. International Journal of Obesity and Related Metabolic Disorders 200327 1472-1478.

10 Ingelson E, Larson MG, Yin X, Wang TJ, Meigs JB, Lipinska I, Benjamin EJ, Keaney JF Jr \& Vasan RS. Circulating ghrelin, leptin and soluble leptin receptor concentrations and cardiometabolic risk factors in a community-based sample. Journal of Clinical Endocrinology and Metabolism 200893 3149-2157.

11 Zambon A, Hashimoto SI \& Brunzell JD. Analysis of techniques to obtain plasma for measurement of levels of free fatty acids. Journal of Lipid Research 199334 1021-1028.

12 Perseghin G, Lattuada G, De Cobelli F, Esposito A, Belloni E, Canu T, Ragogna F, Scifo P, Del Maschio A \& Luzi L. Plasma retinol binding protein-4 (RBP-4), leptin and adiponectin concentrations are related to ectopic fat accumulation. Journal of Clinical Endocrinology and Metabolism 200792 4883-4888.

13 Perseghin G, Burska A, Lattuada G, Alberti G, Costantino F, Ragogna F, Oggionni S, Scollo A, Terruzzi I \& Luzi L. Increased serum resistin in elite endurance athletes with high insulin sensitivity. Diabetologia 200649 1893-1900.

14 Caumo A, Perseghin G, Brunani A \& Luzi L. New insights on the simultaneous assessment of insulin sensitivity and $\beta$-cell function with the HOMA2 method. Diabetes Care $2006292733-2734$.

15 Johnson MM, Chin R \& Haponik EF. Nutrition, respiratory function, and disease. In Modern Nutrition in Health and Disease, pp 1473-1490. Eds ME Shils, JA Olson, M Shike \& AC Ross. Baltimore, MD: Lippincott Williams \& Wilkins, 1999.

16 Frayn KN. Calculation of substrate oxidation rates in vivo from gaseous exchange. Journal of Applied Physiology $1983 \mathbf{5 5}$ 628-634.

17 Bouassida A, Chamari K, Zaouali M, Feki Y, Zbidi A \& Tabka Z. Review on leptin and adiponectin responses and adaptations to acute and chronic exercise. British Journal of Sports Medicine, 2008. In Press DOI: $10.1136 /$ bjsm.2008.046151.

18 Hagobian TA, Sharoff CG, Stephens BR, Wade GN, Enrique Silva J, Chipkin SR \& Braun B. Effects of exercise on energy-regulating hormones and appetite in men and women. American Journal of Physiology. Regulatory, Integrative and Comparative Physiology 2009 296 R233-R242.

19 Befroy DE, Petersen KF, Dufour S, Mason GF, Rothman DL \& Shulman GI. Increased substrate oxidation and mitochondrial uncoupling in skeletal muscle of endurance-trained individuals. PNAS 200828 16701-16706.

20 Graham TE, Yang Q, Blüher M, Hammarstedt A, Ciaraldi TP, Henry RR, Wason CJ, Oberbach A, Jansson PA, Smith U \& Kahn BB. Retinol-binding protein 4 and insulin resistance in lean, obese, and diabetic subjects. New England Journal of Medicine 2006 $3542552-2563$.

Received 20 August 2009

Accepted 14 September 2009 